\title{
The effect of aluminium on enzyme activities in two wheat cultivars
}

\author{
N. Nasr ${ }^{1^{*}}$, J. Carapetian ${ }^{1}$, R. Heidari ${ }^{1}$, S. Asri Rezaei ${ }^{2}$, N. Abbaspour ${ }^{1}$, R. Darvishzadeh ${ }^{3,4}$ and \\ F. Ghezelbash ${ }^{1}$ \\ ${ }^{1}$ Department of Biology, Faculty of Science, Urmia University, Urmia, Iran. \\ ${ }^{2}$ Faculty of Veterinary Medicine, Urmia University, Urmia, Iran. \\ ${ }^{3}$ Institute of Biotechnology, Urmia University, Urmia, Iran. \\ ${ }^{4}$ Department of Agronomy and Plant Breeding, Urmia University, Urmia, Iran.
}

Accepted 9 March, 2011

\begin{abstract}
In order to study the effect of different aluminum (AI) concentrations on the enzyme activities of wheat seedlings and the effect of malate and citrate treatments as chelates for reducing the noxious effect of Al in medium culture, the seedlings of two wheat cultivars, Darab (Al-sensitive) and Maroon (Al-tolerant) were grown on hydroponic solution (non modified Hoagland solution) containing $\mathrm{AlCl}_{3}(0-100-200-300$ $\mu M)$. Factorial experiment was realized in a complete randomized design with three replications. The activity of different enzymes such as ascorbate peroxidase (APX), catalase (CAT), glutathione reductase (GR) and superoxide dismutase (SOD) in root and shoot were measured. Analysis of variance revealed that, the activity of studied enzymes (APX, CAT, GR and SOD) in root and shoot were affected by the main effects of Al concentration. However, in the case of catalase activity in root, the main effect of genotypes as well as genotypexAl concentration was also significant. APX activity in root was not significantly differed between Maroon and Darab in all Al concentrations. But by increasing Al concentration in root medium APX activity was significantly decreased. In the case of SOD activity, we did not find any difference between the studied genotypes in all Al concentrations but its content in roots was affected by the amount of $A$ applied in medium. So that by increasing the amount of Al, SOD content increased in the genotypes similarly. The same trend was observed for catalase activity in root. In the case of GR activity, we did not find any difference between the genotypes in all Al concentrations but its content in root was affected by applying $\mathrm{Al}$ in medium compared with control medium, so that GR content increased in both genotypes similarly. The activity of investigated enzymes showed the same trend in the shoot. The effect of malate and citrate was also studied on reducing the noxious effect of $\mathrm{Al}$ in root. Analysis of variance revealed that, there were significant differences within the treatments on the enzymes activity in root (not in the shoot) except for catalase. However, some interaction effects were significant. This means that malate or citrate application was effective in some Al concentrations.
\end{abstract}

Key words: Maroon (Al tolerant), Darab (Al sensitive), ascorbate peroxidase (APX), catalase (CAT), glutathione reductase (GR), superoxide dismutase (SOD).

\section{INTRODUCTION}

Aluminum (Al) toxicity is a major factor limiting crop

${ }^{*}$ Corresponding author. E-mail: nasr_na2002@yahoo.com. Tel: + 989143885342 . Fax: $+441277670 \overline{7}$. productivity in many acid soils throughout the tropics and subtropics. At mildly acidic or neutral soil $\mathrm{pH}$ values, it occurs primarily as insoluble deposits and essentially is biologically inactive. The acidity of the soil gradually increases as a result of the effects of environmental factors, especially acid rain. Acid soils occupy 30 to $40 \%$ 
of the arable lands (von Uexküll and Mutert, 1995) and as a consequence of a rapid industrial development and environmental pollution, this area increases from year to year especially in developing countries.

Al stress induces the expression of a number of genes; over 20 of them have already been isolated and characterized in wheat (Snowden et al., 1995; Cruz-Ortega et al., 1997; Hamilton et al., 2001), tobacco (Ezaki et al., 1995; 1996) and Arabidopsis (Richards et al., 1998) and their hypothetical functions in Al toxicity or Al resistance mechanisms have been proposed. Since some of these Al-induced genes are coding for antioxidant enzymes (glutathione-S-transferase, peroxidase, superoxide dismutase, ascorbate peroxidase and catalase), it has been suggested that, there is a strong correlation between Al stress and oxidative stress in plants (Cakmak and Horst, 1991; Richards et al., 1998; Simonovicova et al., 2004). A common feature of several stresses (including Al toxicity) is enhanced production of active oxygen species (AOS), which are generally considered harmful to plant cells (Richards et al., 1998; Tamas et al., 2004). However, it has been recently recognized that hydrogen peroxide plays a central role in several physiological processes, such as defense reaction, stomatal closure, programmed cell death, peroxisome biogenesis, cell wall cross-linking and lignin synthesis (Van Breusegem et al., 2001; Neill et al., 2002). More recently, hydrogen peroxide was also identified as an expression of the signal molecule of several genes in plants (Desikan et al., 2000). The key role of antioxidant enzymes is to reduce or scavenge reactive oxygen species, such as superoxide radicals, singlet oxygen, hydrogen peroxide and hydroxyl radicals. The resulting hydrogen peroxide is removed through the activity of the Asada-Halliwell scavenging cycle in chloroplasts and in cytoplasm by ascorbate peroxidase (APX) (Asada, 1992).

Peroxidases (POD) participate in lignin biosynthesis, cell wall cross-linkage, IAA degradation and disease resistance and convert hydrogen peroxide to water (Asada, 1992; Siegel, 1993). The objective of the present study was to assess the role of antioxidant enzymes, including ascorbate peroxidase (APX), superoxide dismutase (SOD), and catalase (CAT) and glutathione reductase (GR) enzymes as possible mechanisms for $\mathrm{Al}$ stress adaptation in wheat.

\section{MATERIALS AND METHODS}

\section{Plant materials and experimental design}

The seeds of two wheat cultivars, Darab (Al sensitive) and Maroon (Al tolerant) were provided from Agricultural Research Center of Karaj. The seeds of two cultivars were sterilized with $5 \%(\mathrm{v} / \mathrm{v})$ sodium hypochlorite for 15 min then, were rinsed with distilled $\mathrm{H}_{2} \mathrm{O}$ for $15 \mathrm{~min}$ and were kept in the dark for $24 \mathrm{~h}$ at $25^{\circ} \mathrm{C}$. Germinated seeds were placed on a plastic net, which was floated on a continuously aerated solution containing $0.5 \mathrm{mM} \mathrm{CaCl}_{2}$. The seedlings were kept in the dark for 1 day at $25^{\circ} \mathrm{C}$ and then were moved to natural light conditions. Solution was renewed daily and seedlings were selected for treatment by measuring uniform root length. Pre-culture solution were replaced by hydroponic solution (non modified Hoagland) containing $\mathrm{AlCl}_{3}(0-100-200-300 \mu \mathrm{M})$ and $\mathrm{pH}$ was kept constant at 4 . Factorial experiment was realized in a complete randomized design with three replications. Each replication consisted of one Petri dish of ten seedlings per cultivar and $\mathrm{AlCl}_{3}$ combinations. Treatment solutions were renewed every 3 days with fresh solution (Zakir et al., 2005). The plants were grown for 15 days under a $16 \mathrm{~h}$ photoperiod. Then, 15 days old plants were used for the experiments which in citrate and malate were used as phytochelator for decreasing the effect of aluminum toxicity.

\section{Chlorophyll measurement}

Chlorophyll content of leaves was determined according to Wintermans and De Mots (1965). Briefly, after extraction in 96\% $(\mathrm{v} / \mathrm{v})$ ethanol, the absorption was measured in different wavelengths depend on the type of chlorophyll (Kuo and Kao, 2003).

\section{Determination of enzymes activities}

For extraction of enzymes, $0.5 \mathrm{~g}$ of fresh weight was homogenized with $0.1 \mathrm{M}$ phosphate buffer $(\mathrm{pH}$ 6.8) in a chilled pestle. The homogenate was centrifuged at $8000 \times \mathrm{g}$ for $20 \mathrm{~min}$ and the supernatant was used for determining of enzymes activities. The whole extraction procedure was carried out at $4^{\circ} \mathrm{C}$. CAT activity was determined by measuring the initial rate of disappearance of $\mathrm{H}_{2} \mathrm{O}_{2}$ (Kato and Shimidzu, 1987). The decrease in $\mathrm{H}_{2} \mathrm{O}_{2}$ was followed as the decline in absorbance at $240 \mathrm{~nm}$. One unite of CAT was defined as the amount of enzyme which breaks down $1 \mathrm{nmol}$ $\mathrm{H}_{2} \mathrm{O}_{2}$ per min. Superoxide dismutase (SOD) was determined according to Paoliett et al. (1986). One unit of SOD was defined as the amount of enzyme which inhibits the rate of NADH oxidation by $50 \%$ compared with blank. Ascorbate peroxidase (APX) activity was determined according to Nakano and Asada (1981). The decreased in ascorbate concentration was followed at $290 \mathrm{~nm}$. One unit of APX was defined as the amount of enzyme which breaks down 1 $\mu \mathrm{mol}$ of ascorbate per min. Finally, glutathione reductase (GR) was defined as the amount of enzyme which decreases $A_{340}(1 \mathrm{u}$ per $\mathrm{min})$. All of the earlier mentioned experiments were repeated three times (Kuo and Kao, 2003).

\section{Data analysis}

Analysis of variance was performed using the general linear model (GLM) procedure in the SAS software (SAS Institute Inc., Cary, NC, USA). The main effects of genotype, Al concentration as well as their interaction were determined. To generate a trend analysis, the Proc REG procedure of PC-SAS is specified (SAS Institute Inc., Cary, NC, USA). Commands for each model are placed after the Proc Reg statement. A separate model statement was required for linear, quadratic and cubic trends.

\section{RESULTS}

Analysis of variance revealed that, the activity of enzymes such as ascorbate peroxidase (APX), catalase, glutathione reductase (GR) and superoxide dismutase (SOD) in root and shoot were affected by the main effect 
Table 1. Analysis of variance summary for enzymes data under different Al concentrations. Data were analyzed using procedures for a completely randomized design.

\begin{tabular}{|c|c|c|c|c|c|c|c|c|c|}
\hline \multirow{2}{*}{\multicolumn{2}{|c|}{ Source }} & \multirow[b]{2}{*}{ DF } & \multicolumn{4}{|c|}{ Mean of square } & \multirow[b]{2}{*}{$\begin{array}{c}\text { Chlorophyll } \\
\text { A }\end{array}$} & \multirow[b]{2}{*}{$\begin{array}{c}\text { Chlorophyll } \\
\text { B }\end{array}$} & \multirow[b]{2}{*}{ Caretenoid } \\
\hline & & & APX-S & Catalase-S & GR-S & SOD-S & & & \\
\hline Line & & 1 & $0.003^{\text {ns }}$ & $21.08^{\text {ns }}$ & $0.00003^{\mathrm{ns}}$ & $0.05^{\mathrm{ns}}$ & $4.78^{\text {ns }}$ & $0.036^{\text {ns }}$ & $2554.41^{\text {ns }}$ \\
\hline Al concentration & & 3 & $175.53^{\star *}$ & $2102.81^{\star \star}$ & $0.02^{* *}$ & $99.60^{* *}$ & $4.76^{*}$ & $0.023^{\text {ns }}$ & $58507.74^{*}$ \\
\hline $\begin{array}{l}\text { Line } \\
\text { concentration }\end{array}$ & $\times \mathrm{Al}$ & 3 & $6.70^{\mathrm{ns}}$ & $172.31^{\mathrm{ns}}$ & $0.0008^{\text {ns }}$ & $4.12^{\mathrm{ns}}$ & $3.79^{\text {ns }}$ & $0.035^{*}$ & $48182.51^{*}$ \\
\hline Error & & 16 & 4.88 & 75.07 & 0.0004 & 2.72 & 1.22 & 0.009 & 13328.22 \\
\hline C.V. & & & 8.17 & 25.41 & 7.61 & 5.80 & 27.43 & 23.86 & 32.50 \\
\hline
\end{tabular}

Table 1. Continued.

\begin{tabular}{lccccc}
\hline \multirow{2}{*}{ Source } & \multirow{2}{*}{ DF } & \multicolumn{4}{c}{ Mean of square } \\
\cline { 3 - 6 } & & APX-R & Catalase-R & GR-R & SOD-R \\
\hline Line & 1 & $18.69^{\text {ns }}$ & $6253.30^{* *}$ & $0.002^{\text {ns }}$ & $10.51^{\text {ns }}$ \\
Al concentration & 3 & $176.44^{* *}$ & $16370.70^{* *}$ & $0.02^{* *}$ & $99.25^{* *}$ \\
Line $\times$ Al concentration & 3 & $9.02^{\text {ns }}$ & $1124.86^{* *}$ & $0.0009^{\text {ns }}$ & $5.07^{\text {ns }}$ \\
Error & 14 & 7.17 & 194.52 & 0.0006 & 4.04 \\
C.V. & & 15.07 & 13.20 & 6.86 & 5.67 \\
\hline
\end{tabular}

CV: Coefficient of variation. $\mathrm{df}=$ Degrees of freedom; **, *: Significant at 0.01 and 0.05 probability level; ns: non significant.

of $\mathrm{Al}$ concentration. Only concerning to catalase activity in root, the main effect of genotype as well as genotype $x$ Al concentration was significant (Table 1). As shown in Figure 1, the APX activity in root was not significantly different between Maroon and Darab in all studied Al concentrations. However, by increasing the Al concentration in medium, APX activity in root was significantly decreased. In the case of SOD activity, there was no difference between both studied genotypes in all applied Al concentrations. However, its content was affected by the amount of $\mathrm{Al}$ concentration. Such that by increasing the amount of $\mathrm{Al}$ its content increased in the genotypes similarly. The same trend was observed for the catalase activity in root. As for superoxide dismutase, there was no difference between two studied genotypes in all applied Al concentrations from the view point of GR activity. But in comparison to control plants glutathione reductase activity in roots was affected by applying $\mathrm{Al}$ in medium and its content increased in two genotypes similarly. The activity of the investigated enzymes showed the same trend in the shoots.

A separate regression analysis considering $\mathrm{Al}$ concentration as an independent variable and the enzymes activity as a dependent variable was conducted for each genotype. The activity of APX in Maroon and Darab root best fit the cubic and linear model, respectively, as indicated by a significant T-value (Table 2). This means that, activity of APX in root of these genotypes followed the different basic trend. $R^{2}$ values for Maroon and Darab were 0.99 and 0.61 , respectively. This also means that, 99 and $61 \%$ of the variation was explained by the model. These values are high because $R^{2}$ values for biological data generally range from 0.50 to 0.90 , whereas, a low $R^{2}$ for non-biological data may be 0.90 (Kleinbaum and Kupper, 1978). In contrast, analysis of APX activity in shoot using polynomial contrasts indicated that, the response of Maroon and Darab seedlings best fit the linear and cubic model. Concerning to other enzymes we also found a difference in fitted model between stem and root (Table 2).

We have also studied the effect of genotype and $\mathrm{Al}$ concentration on the chlorophyll-A, chlorophyll-B and caretenoid content. Analysis of variance for chlorophyllcontent revealed that, only the main effect of $\mathrm{Al}$ concentration was significant. In the case of chlorophyll-B content, the interaction between genotype and $\mathrm{Al}$ concentration was significant. For caretenoid both the main effect of Al concentration and the interaction effect between genotype and $\mathrm{Al}$ concentration were significant. In the second experiment, the effect of citrate and malate treatments was studied on decreasing the noxious effect of $\mathrm{Al}$ in medium. Analysis of variance showed that, there were significant differences within applied treatments on studied enzymes activities in root not in the shoot except for catalase activity (Table 3). However, some interaction effects were significant. This means that, the effect of 

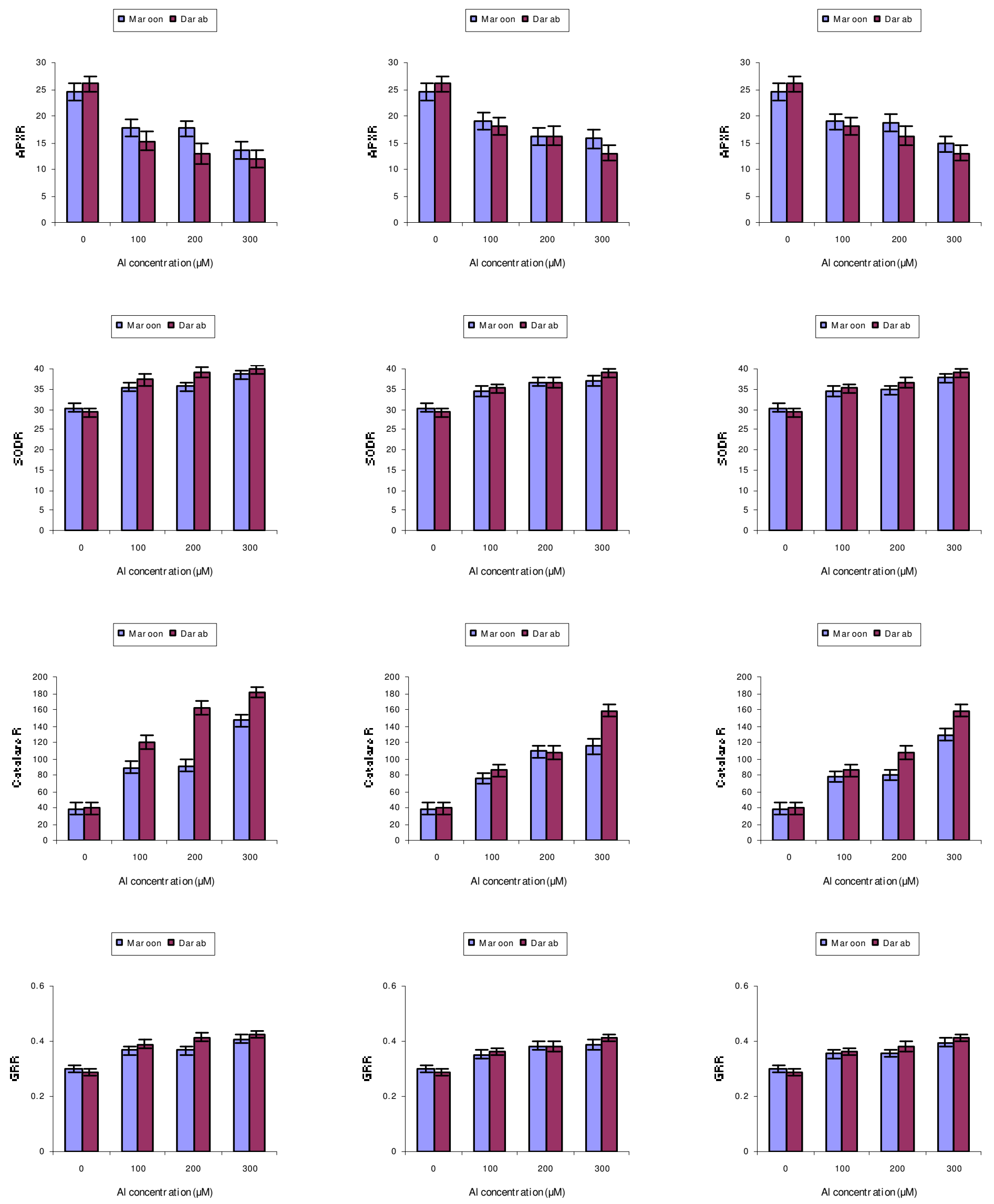

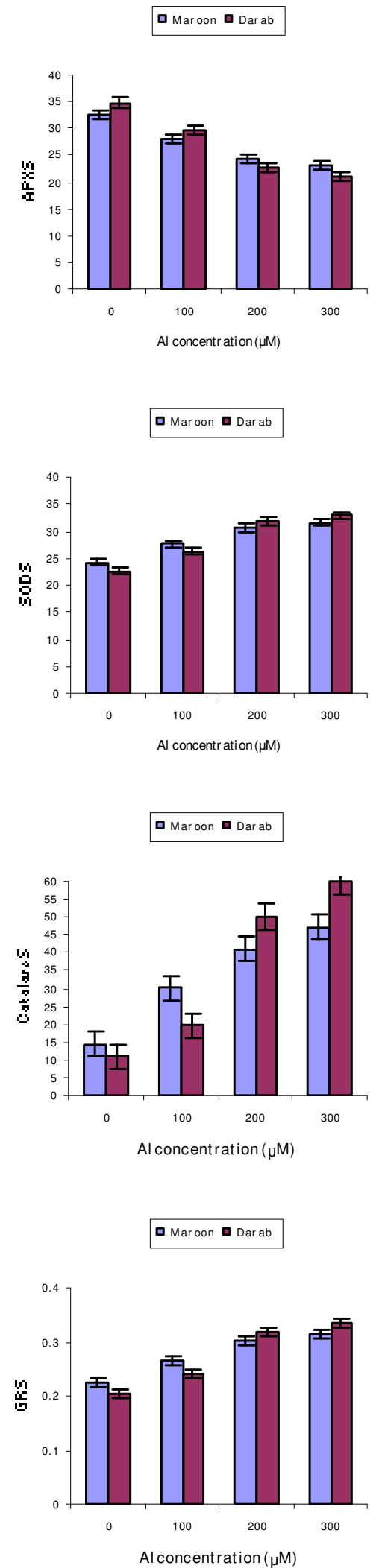
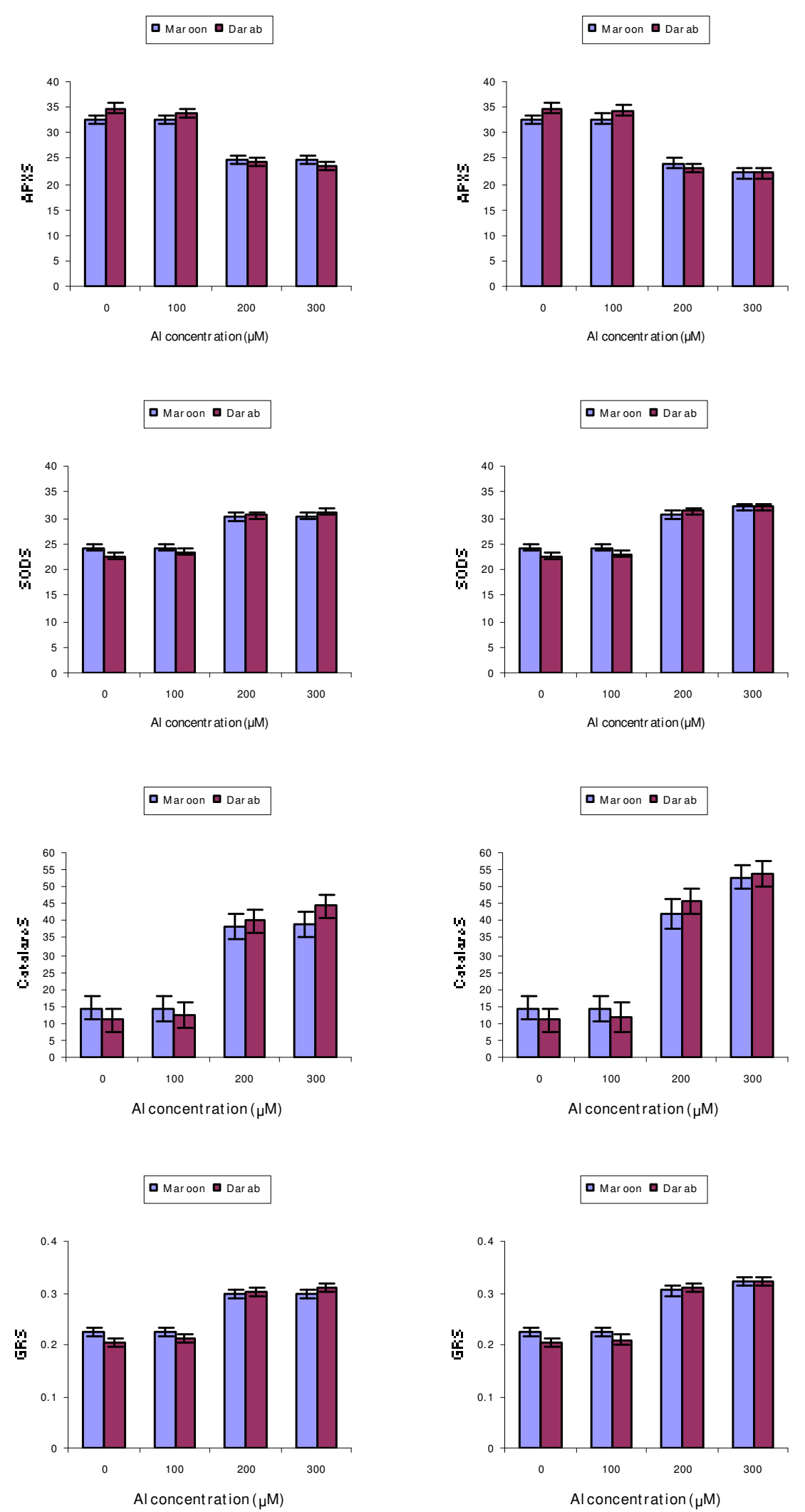

Figure 1. Effect of malate and citrate treatments on reducing the noxious effect of $\mathrm{Al}$ in medium culture. The first column from left show the effect of just different Al concentrations in medium culture on the different enzyme activities. The second column show the effect of just Al concentrations in medium culture together with the malate on the different enzyme activities and the third column show the effect of $\mathrm{Al}$ concentrations in medium culture together with the citrate on the different enzyme activities. 
Table 2. Summary table for wheat enzymes data in different Al concentrations using regression analysis.

\begin{tabular}{|c|c|c|c|c|c|c|c|c|c|c|c|c|c|c|}
\hline \multirow{2}{*}{ Character } & \multirow{2}{*}{ Line } & \multirow{2}{*}{ Source } & \multicolumn{4}{|l|}{ Linear } & \multicolumn{4}{|c|}{ Quadratic } & \multicolumn{4}{|l|}{ Cubic } \\
\hline & & & $\operatorname{Pr}>|T|^{a}$ & $\mathbf{R}^{2}$ & Estimate & SE & $\operatorname{Pr}>|\mathrm{T}|$ & $\mathbf{R}^{2}$ & Estimate & SE & $\operatorname{Pr}>|\mathrm{T}|$ & $\mathbf{R}^{2}$ & Estimate & SE \\
\hline \multirow{8}{*}{ APX-R } & \multirow[t]{4}{*}{$\mathrm{R}$} & Intercept & - & - & 23.35 & 0.75 & - & - & 24.06 & 0.80 & - & - & 24.58 & 0.28 \\
\hline & & Concentration & ** & 0.86 & -0.03 & & ** & - & -0.05 & 0.01 & ** & - & -0.14 & 0.01 \\
\hline & & Concentration ${ }^{2}$ & - & - & - & - & ns & 0.88 & 0.00007 & 0.00004 & ** & - & 0.0009 & 0.0001 \\
\hline & & Concentration $^{3}$ & - & - & - & - & - & - & - & - & ** & 0.99 & -0.000002 & $2.107168 \mathrm{E}-7$ \\
\hline & \multirow[t]{4}{*}{$\mathrm{S}$} & Intercept & - & - & 23.85 & 2.26 & - & - & 25.81 & 2.18 & \multirow[t]{4}{*}{-} & - & 26.04 & 2.34 \\
\hline & & Concentration & ** & 0.61 & -0.05 & 0.01 & * & - & -0.12 & 0.04 & & - & -0.17 & 0.11 \\
\hline & & Concentration $^{2}$ & - & - & - & - & ns & 0.71 & 0.0003 & 0.0001 & & - & 0.0007 & 0.001 \\
\hline & & Concentration ${ }^{3}$ & - & - & - & - & - & - & - & - & & 0.68 & -0.000001 & 0.000002 \\
\hline \multirow{8}{*}{ APX-S } & \multirow[t]{4}{*}{$\mathrm{R}$} & Intercept & - & - & 31.99 & 1.41 & - & - & 32.82 & 1.64 & - & - & 32.70 & 1.78 \\
\hline & & Concentration & $* *$ & 0.63 & -0.03 & 0.008 & ns & - & -0.06 & 0.03 & ns & - & -0.04 & 0.07 \\
\hline & & Concentration $^{2}$ & - & - & - & - & ns & 0.62 & 0.00008 & 0.00008 & ns & - & -0.00009 & 0.0006 \\
\hline & & Concentration $^{3}$ & - & - & - & - & - & - & - & - & ns & 0.58 & $3.915 \mathrm{E}-7$ & 0.000001 \\
\hline & \multirow[t]{4}{*}{$S$} & Intercept & - & - & 34.33 & 0.71 & - & - & 35.24 & 0.63 & - & - & 34.84 & 0.30 \\
\hline & & Concentration & ** & 0.94 & -0.05 & 0.004 & ** & - & -0.08 & 0.01 & ns & - & -0.01 & 0.01 \\
\hline & & Concentration $^{2}$ & - & - & - & - & * & 0.96 & 0.00009 & 0.00003 & ** & - & -0.0005 & 0.0001 \\
\hline & & Concentration $^{3}$ & - & - & - & - & - & - & - & & $* *$ & 0.99 & 0.000001 & $2.227478 \mathrm{E}-7$ \\
\hline \multirow{9}{*}{ Caretenoid } & \multirow[t]{4}{*}{$\mathrm{R}$} & Intercept & - & - & 354.95 & 27.73 & - & - & 355.86 & 34.04 & - & - & 356.91 & 37.008 \\
\hline & & Concentration & ns & 0.02 & -0.07 & 0.15 & ns & - & -0.09 & 0.55 & ns & - & -0.26 & 1.42 \\
\hline & & Concentration $^{2}$ & - & - & - & - & ns & 0.02 & 0.00009 & 0.002 & ns & - & 0.002 & 0.01 \\
\hline & & Concentration $^{3}$ & - & - & - & - & - & - & - & - & ns & 0.02 & -0.000004 & 0.00003 \\
\hline & & & & & & & & & & & & & & \\
\hline & \multirow[t]{4}{*}{$S$} & Intercept & - & - & 571.75 & 71.02 & - & - & 592.47 & 86.17 & - & - & 615.07 & 86.69 \\
\hline & & Concentration & ** & 0.52 & -1.37 & 0.38 & ns & - & -2.00 & 1.38 & ns & - & -5.54 & 3.33 \\
\hline & & Concentration $^{2}$ & - & - & - & - & ns & 0.48 & 0.002 & 0.004 & ns & - & 0.04 & 0.03 \\
\hline & & Concentration $^{3}$ & - & - & - & - & - & - & - & - & ns & 0.50 & -0.00008 & 0.00007 \\
\hline
\end{tabular}

\begin{tabular}{|c|c|c|c|c|c|c|c|c|c|c|c|c|c|c|}
\hline \multirow{2}{*}{ Character } & \multirow{2}{*}{ Line } & \multirow{2}{*}{ Source } & \multicolumn{4}{|l|}{ Linear } & \multicolumn{4}{|c|}{ Quadratic } & \multicolumn{4}{|l|}{ Cubic } \\
\hline & & & $\operatorname{Pr}>|\mathrm{T}|^{\mathrm{a}}$ & $\mathbf{R}^{2}$ & Estimate & SE & $\mathrm{Pr}>|\mathrm{T}|$ & $\mathbf{R}^{2}$ & Estimate & SE & $\operatorname{Pr}>|\mathrm{T}|$ & $\mathbf{R}^{2}$ & Estimate & SE \\
\hline Catalase-R & $\mathrm{R}$ & Intercept & - & - & 42.56 & 6.46 & - & - & 43.99 & 7.88 & - & - & 38.91 & 3.00 \\
\hline
\end{tabular}


Table 2. Contd.

\begin{tabular}{|c|c|c|c|c|c|c|c|c|c|c|c|c|c|c|}
\hline & & Concentration & $* *$ & 0.88 & 0.33 & 0.04 & ns & - & 0.28 & 0.13 & $* \star$ & - & 1.08 & 0.12 \\
\hline & & Concentration $^{2}$ & - & - & - & - & ns & 0.88 & 0.0001 & 0.0004 & $* *$ & - & -0.008 & 0.001 \\
\hline & & Concentration $^{3}$ & - & - & - & - & - & - & - & - & $* *$ & 0.98 & 0.00002 & 0.000002 \\
\hline & \multirow[t]{4}{*}{$\mathrm{S}$} & Intercept & - & - & 52.19 & 12.42 & - & - & 39.78 & 10.78 & - & - & 39.31 & 11.80 \\
\hline & & Concentration & $* *$ & 0.85 & 0.47 & 0.07 & ** & - & 0.94 & 0.19 & ns & - & 1.04 & 0.53 \\
\hline & & Concentration $^{2}$ & - & - & - & - & * & 0.91 & -0.002 & 0.0006 & ns & - & -0.003 & 0.005 \\
\hline & & Concentration $^{3}$ & - & - & - & - & - & - & - & - & ns & 090 & 0.000002 & 0.00001 \\
\hline & $\mathrm{R}$ & Intercept & - & - & 16.75 & 5.39 & - & - & 14.40 & 6.44 & - & - & 14.40 & 7.01 \\
\hline & & Concentration & $\star *$ & 0.55 & 0.11 & 0.03 & ns & - & 0.18 & 0.10 & ns & - & 0.18 & 0.27 \\
\hline & & Concentration $^{2}$ & - & - & - & - & ns & 0.53 & -0.0002 & 0.0003 & ns & - & -0.0002 & 0.002 \\
\hline & & Concentration $^{3}$ & - & - & - & - & - & - & - & - & ns & 0.47 & $-2.58889 E-8$ & 0.000005 \\
\hline \multirow[t]{8}{*}{ Catalase-S } & $\mathrm{S}$ & Intercept & - & - & 8.50 & 2.62 & - & - & 8.79 & 3.22 & - & - & 10.91 & 0.99 \\
\hline & & Concentration & $* *$ & 0.94 & 0.18 & 0.01 & ** & - & 0.17 & 0.05 & $\star *$ & - & -0.16 & 0.04 \\
\hline & & Concentration $^{2}$ & - & - & - & - & ns & 0.93 & 0.00003 & 0.0002 & $* *$ & - & 0.003 & 0.0003 \\
\hline & & Concentration ${ }^{3}$ & - & - & - & - & - & - & - & & $* *$ & 0.99 & -0.000007 & $7.361438 \mathrm{E}-7$ \\
\hline & $\mathrm{R}$ & Intercept & - & - & 4.60 & 0.39 & - & - & 4.34 & 0.45 & - & - & 4.33 & 0.49 \\
\hline & & Concentration & ns & 0.02 & -0.0009 & 0.002 & ns & - & 0.007 & 0.007 & ns & - & 0.009 & 0.02 \\
\hline & & Concentration $^{2}$ & - & - & - & - & ns & 0.13 & -0.00003 & 0.00002 & ns & - & -0.00005 & 0.0002 \\
\hline & & Concentration $^{3}$ & - & - & - & - & - & - & - & - & ns & 0.14 & $5.5 \mathrm{E}-8$ & $3.681328 \mathrm{E}-7$ \\
\hline \multicolumn{15}{|c|}{ Chlorophyll A } \\
\hline & $\mathrm{S}$ & Intercept & - & - & 5.50 & 0.57 & - & - & 5.56 & 0.70 & - & - & 5.59 & 0.75 \\
\hline & & Concentration & $* *$ & 0.61 & -0.01 & 0.003 & ns & - & -0.02 & 0.01 & ns & - & -0.02 & 0.03 \\
\hline & & Concentration $^{2}$ & - & - & - & - & ns & 0.56 & 0.000006 & 0.00004 & ns & - & 0.00005 & 0.0003 \\
\hline & & Concentration $^{3}$ & - & - & - & - & - & - & - & - & ns & 0.51 & $-9.46111 E-8$ & 5.624392E-7 \\
\hline
\end{tabular}

\begin{tabular}{|c|c|c|c|c|c|c|c|c|c|c|c|c|c|c|}
\hline \multirow{2}{*}{ Character } & \multirow{2}{*}{ Line } & \multirow{2}{*}{ Source } & \multicolumn{4}{|l|}{ Linear } & \multicolumn{4}{|c|}{ Quadratic } & \multicolumn{4}{|l|}{ Cubic } \\
\hline & & & $\operatorname{Pr}>|T|^{a}$ & $\mathbf{R}^{2}$ & Estimate & SE & $\operatorname{Pr}>|\mathrm{T}|$ & $\mathbf{R}^{2}$ & Estimate & SE & $\operatorname{Pr}>|\mathrm{T}|$ & $\mathbf{R}^{2}$ & Estimate & SE \\
\hline \multirow{4}{*}{$\begin{array}{l}\text { Chlorophyll } \\
\text { B }\end{array}$} & $R$ & Intercept & - & - & 0.41 & 0.04 & - & - & 0.41 & 0.04 & - & - & 0.42 & 0.04 \\
\hline & & Concentration & ns & 0.06 & 0.0001 & 0.0002 & ns & - & 0.00008 & 0.0007 & ns & - & -0.001 & 0.002 \\
\hline & & Concentration $^{2}$ & - & - & - & - & ns & 0.06 & $2.155602 \mathrm{E}-7$ & 0.000002 & ns & - & 0.00001 & 0.00002 \\
\hline & & Concentration $^{3}$ & - & - & - & - & - & - & - & & ns & 0.12 & $-2.4376 \mathrm{E}-8$ & $3.331914 \mathrm{E}-8$ \\
\hline
\end{tabular}


Table 2. Contd.

\begin{tabular}{|c|c|c|c|c|c|c|c|c|c|c|c|c|c|c|c|c|}
\hline & \multirow[t]{4}{*}{ S } & Intercept & - & - & 0.48 & 0.06 & - & - & 0.41 & \multicolumn{2}{|l|}{0.06} & - & \multicolumn{2}{|c|}{-} & 0.42 & 0.06 \\
\hline & & Concentration & * & 0.36 & -0.0009 & 0.0003 & ns & - & 0.001 & \multicolumn{2}{|l|}{0.0009} & $\mathrm{n}$ & \multicolumn{2}{|c|}{-} & 0.0002 & 0.002 \\
\hline & & Concentration $^{2}$ & - & - & - & - & * & 0.55 & -0.000007 & \multicolumn{2}{|c|}{0.000003} & $\mathrm{n}$ & \multicolumn{2}{|c|}{-} & 0.000003 & 0.00002 \\
\hline & & Concentration $^{3}$ & - & - & \multicolumn{2}{|c|}{-} & - & - & - & \multicolumn{2}{|l|}{-} & $\mathrm{n}$ & \multicolumn{2}{|r|}{0.51} & $-2.17434 \mathrm{E}-8$ & 4.650606E-8 \\
\hline & \multirow[t]{4}{*}{$\mathrm{R}$} & Intercept & - & - & 0.31 & 0.007 & - & - & 0.31 & \multicolumn{2}{|l|}{0.008} & - & \multicolumn{2}{|c|}{ 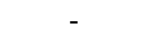 } & 0.30 & 0.003 \\
\hline & & Concentration & ** & 0.86 & 0.0003 & 0.00004 & ** & - & 0.0005 & 0.0001 & & ** & . & - & 0.001 & 0.0001 \\
\hline & & Concentration $^{2}$ & - & - & - & - & ns & 0.88 & $-6.58333 \mathrm{E}-7$ & 4.11503 & $2 \mathrm{E}-7$ & ** & & - & -0.000009 & $9.691061 \mathrm{E}-7$ \\
\hline & & Concentration $^{3}$ & - & - & - & - & - & - & - & - & & ** & & 0.99 & $1.738889 \mathrm{E}-8$ & $2.130032 \mathrm{E}-9$ \\
\hline GR-R & & & & & & & & & & & & & & & & \\
\hline & $S$ & Intercept & - & - & 0.31 & 0.02 & - & - & 0.29 & 0.02 & & - & & - & 0.29 & 0.02 \\
\hline & & Concentration & ** & 0.62 & 0.0005 & 0.0001 & * & - & 0.001 & 0.0004 & & $\mathrm{n}$ & & - & 0.002 & 0.001 \\
\hline & & Concentration $^{2}$ & - & - & - & - & ns & 0.73 & -0.000002 & 0.00000 & & $\mathrm{n}$ & & - & -0.000007 & 0.00001 \\
\hline & & Concentration $^{3}$ & - & - & - & - & - & - & - & & & $\mathrm{n}$ & & 0.70 & $1.055556 \mathrm{E}-8$ & $1.96756 \mathrm{E}-8$ \\
\hline & $\mathrm{R}$ & Intercept & - & - & 0.23 & 0.01 & - & - & 0.22 & 0.02 & & - & & - & 0.22 & 0.02 \\
\hline & & Concentration & $* *$ & 0.62 & 0.0003 & 0.00007 & ns & - & 0.0005 & 0.0003 & & $\mathrm{n}$ & & - & 0.0004 & 0.0007 \\
\hline & & Concentration $^{2}$ & - & - & - & - & ns & 0.62 & -7.66667E-7 & 7.96942 & $5 \mathrm{E}-7$ & $\mathrm{n}$ & & - & 8.833333E-7 & 0.000006 \\
\hline & & Concentration $^{3}$ & - & - & - & - & - & - & - & - & & $\mathrm{n}$ & & 0.58 & $-3.66667 E-9$ & $1.25339 \mathrm{E}-8$ \\
\hline GR-S & & & & & & & & & & & & & & & & \\
\hline & $S$ & Intercept & - & - & 0.21 & 0.007 & - & - & 0.20 & 0.008 & & - & & - & 0.20 & 0.003 \\
\hline & & Concentration & ** & 0.93 & 0.0005 & 0.00004 & ** & - & 0.0006 & 0.0001 & & ** & & - & -0.0002 & 0.0001 \\
\hline & & Concentration $^{2}$ & - & - & - & - & ns & 0.93 & $-5.83333 \mathrm{E}-7$ & 4.1800 & $3 E-7$ & ** & & - & 0.000007 & 0.000001 \\
\hline & & Concentration $^{3}$ & - & - & - & - & - & - & - & - & & ** & & 0.98 & $-1.73333 \mathrm{E}-8$ & 2.475185E-9 \\
\hline & Line & & Linear & & & & Quadra & & & & Cubi & & & & & \\
\hline Character & LIne & Source & $\mathrm{Pr}>|\mathrm{T}|^{\mathrm{a}}$ & $\mathbf{R}^{2}$ & Estimate & SE & $\operatorname{Pr}>|\mathrm{T}|$ & $\mathbf{R}^{2}$ & Estimate & SE & Pr>17 & & $\mathbf{R}^{2}$ & & timate & E \\
\hline SOD-R & $\mathrm{R}$ & Intercept & - & - & 31.24 & 0.56 & - & - & 30.71 & 0.60 & - & & - & & .32 & .21 \\
\hline & & Concentration & ** & 0.86 & 0.03 & 0.003 & ** & - & 0.04 & 0.01 & ** & & - & 0. & & .008 \\
\hline & & Concentration $^{2}$ & - & - & - & - & ns & 0.88 & -0.00005 & 0.00003 & $\star *$ & & - & & 0006 & .00007 \\
\hline & & Concentration $^{3}$ & - & - & - & - & - & - & - & - & ** & & 0.98 & & 00001 & $.580746 \mathrm{E}-7$ \\
\hline & $\mathrm{S}$ & Intercept & - & - & 30.86 & 1.70 & - & - & 29.39 & 1.63 & - & & - & & .22 & .76 \\
\hline & & Concentration & $* *$ & 0.61 & 0.03 & 0.009 & * & - & 0.09 & 0.03 & ns & & - & 0. & & .08 \\
\hline & & Concentration $^{2}$ & - & - & - & - & ns & 0.71 & -0.0001 & 0.00009 & ns & & - & & 0006 & .0007 \\
\hline
\end{tabular}


Table 2. Contd.

\begin{tabular}{|c|c|c|c|c|c|c|c|c|c|c|c|c|c|c|}
\hline & & Concentration $^{3}$ & - & - & - & - & - & - & - & - & ns & 0.68 & $8.286111 \mathrm{E}-7$ & 0.000002 \\
\hline \multirow[t]{8}{*}{ SOD-S } & $\mathrm{R}$ & Intercept & - & - & 24.76 & 1.06 & - & - & 24.13 & & - & - & 24.22 & 1.33 \\
\hline & & Concentration & ** & 0.63 & 0.03 & 0.006 & ns & - & 0.04 & & ns & - & 0.03 & 0.05 \\
\hline & & Concentration $^{2}$ & - & - & - & - & ns & 0.63 & -0.00006 & & ns & - & 0.00007 & 0.0005 \\
\hline & & Concentration $^{3}$ & - & - & - & - & - & - & - & - & ns & 0.58 & $-2.93944 \mathrm{E}-7$ & $9.940348 \mathrm{E}-7$ \\
\hline & $\mathrm{S}$ & Intercept & - & - & 22.89 & 0.53 & - & - & 22.28 & 0.52 & - & - & 22.62 & 0.18 \\
\hline & & Concentration & ** & 0.94 & 0.04 & 0.003 & $* *$ & - & 0.06 & 0.008 & ns & - & 0.002 & 0.007 \\
\hline & & Concentration $^{2}$ & - & - & - & - & * & 0.96 & -0.00006 & 0.00003 & ** & - & 0.0005 & 0.00006 \\
\hline & & Concentration $^{3}$ & - & - & - & - & - & - & - & & ** & 0.99 & -0.000001 & SE \\
\hline
\end{tabular}

malate or citrate application in some Al concentrations is effective compared with other Al concentrations. As shown in Figure 1, the application of citrate and malate approximately improved enzymes activities; however, their applications did not change the observed trends (Figure 1).

\section{DISCUSSION}

A uniform increase in superoxide dismutase (SOD), catalase (CAT) and glutathione reductase (GR) activities associated with a gradual decrease in APX activity was detected by increasing $\mathrm{Al}$ concentration in medium culture. SOD is an important enzyme that is associated with antioxidative stress in plants (Guo et al., 2004). Increased superoxide dismutase activity may be due to an elevated content of superoxide radical. It has been shown that, Al enhanced SOD activity in root tips of soybean (Cakmak and Horst, 1991), roots of Arabidopsis (Richards et al., 1998), and roots of sorghum (Peixoto et al., 1999). Lee et al. (2001) suggested that, enhanced activity of superoxide dismutase may function in signaling of oxidative stress and hence, leads to the induction of antioxidant enzymes associated with an $\mathrm{H}_{2} \mathrm{O}_{2}$ scavenging system, particularly an ascorbateglutathione cycle. Al-induced high activity of SOD that was observed in the present study is consistent with previous results obtained from different plants (Boscolo et al., 2003; Tamas et al., 2003; Meriga et al., 2004; Sharma and Dubey, 2007).

The marked increase of catalase activity with increasing Al levels in Maroon and Darab varieties may indicate enhanced production of reactive oxygen species (ROS) under an excess of aluminium (Al). This enhanced activity seems to be related to increased oxidative stress tolerance (Allen, 1995). Among the enzymatic systems considered to play an important role in the cellular defense strategy against oxidative stress, catalase (CAT) plays an important role as it decomposes $\mathrm{H}_{2} \mathrm{O}_{2}$ to water and $\mathrm{O}_{2}$. It has been previously suggested that, accumulation of $\mathrm{H}_{2} \mathrm{O}_{2}$ caused by various environmental stresses would result in the enhanced activity of catalase in order to protect plant cells (Mizuno et al., 1988).

Superoxide dismutase (SOD) and catalase may cooperatively contribute to suppress the lipid peroxidation caused by $\mathrm{H}_{2} \mathrm{O}_{2}$ and diamide as well as aluminium stress in Andropogon and Miscanthus.
Hydrogen peroxide which is cytotoxic and acts both as an oxidant and reductant is detoxified by catalase activity.

$\mathrm{Al}$, in the present study, decreased APX activity in root and shoot of both studied genotypes. Reports available on effect of $\mathrm{Al}$ on ascorbate peroxidase activity have been some what contradictory. Aluminium has been shown to enhance activity of APX in Cucurbita pepo (Dipierro et al., 2005) and rice (Sharma and Dubey, 2007). Activity of chloroplastic-APX in rice, however, was inhibited by Al (Sharma and Dubey, 2007).

Interestingly, the greater catalase activity in Darab as an Al-sensitive genotype than in Maroon as an Al-resistant may be attributed to higher oxidative stress in Darab. Differences in response of Al-resistant in relation to Al-sensitive plant genotypes with respect to activities of antioxidant enzymes have been recorded. For instance, Alresistant genotypes of wheat (Darko et al., 2004) or rice (Ma et al., 2007) showed significantly higher catalase and ascorbate peroxidase activities than that of Al-sensitive counterparts under Al- treatments. The opposite, however, was true for barley (Tamas et al., 2003) and maize (Boscolo et al., 2003) that registered a greater 
Table 3. Analysis of variance summary for wheat enzymes data under different Al concentrations as well as malate and citrate treatments. Data were analyzed using procedures for a completely randomized design.

\begin{tabular}{|c|c|c|c|c|c|}
\hline \multirow{2}{*}{ Source } & \multirow{2}{*}{ DF } & \multicolumn{4}{|c|}{ Mean of square } \\
\hline & & APXs & SODs & Catalases & GRs \\
\hline Genotype & 1 & $2.78^{\text {ns }}$ & $1.82^{\text {ns }}$ & $8.16^{\text {ns }}$ & $0.0003^{\mathrm{ns}}$ \\
\hline Al concentration & 3 & $547.71^{* *}$ & $309.62^{* *}$ & $5911.81^{* *}$ & $0.05^{\star *}$ \\
\hline Treatment & 2 & $21.76^{\star *}$ & $11.59^{\star *}$ & $330.50^{\star *}$ & $0.002^{* *}$ \\
\hline genotype $\times$ treatment & 2 & $0.72^{\mathrm{ns}}$ & $0.30^{\text {ns }}$ & $6.74^{\mathrm{ns}}$ & $0.00003^{\text {ns }}$ \\
\hline Al concentration $\times$ treatment & 6 & $8.75^{\star \star}$ & $4.54^{\star *}$ & $90.31^{*}$ & $0.0006^{*}$ \\
\hline genotype $\times$ Al concentration & 3 & $11.27^{* *}$ & $6.54^{\star *}$ & $141.55^{\star}$ & $0.001^{* *}$ \\
\hline genotype $\times \mathrm{Al}$ concentration $\times$ treatment & 6 & $0.45^{\mathrm{ns}}$ & $0.32^{\mathrm{ns}}$ & $32.09^{\text {ns }}$ & $0.00008^{\text {ns }}$ \\
\hline Error & 46 & 2.20 & 1.23 & 37.91 & 0.0002 \\
\hline $\mathrm{CV}$ & & 5.29 & 4.00 & 20.13 & 5.28 \\
\hline
\end{tabular}

Table 3. Continued.

\begin{tabular}{llllll}
\hline \multirow{2}{*}{ Source } & \multirow{2}{*}{ DF } & \multicolumn{2}{l}{ Mean of square } \\
\cline { 3 - 6 } & & APXr & SODr & Catalaser & GRr \\
\hline Genotype & 1 & $19.40^{\text {ns }}$ & $10.91^{\text {ns }}$ & $7344.42^{* *}$ & $0.002^{\text {ns }}$ \\
Al concentration & 3 & $433.56^{\star *}$ & $243.87^{* *}$ & $35592.00^{* *}$ & $0.04^{\star *}$ \\
Treatment & 2 & $11.20^{\text {ns }}$ & $6.30^{\text {ns }}$ & $2407.24^{* *}$ & $0.001^{\text {ns }}$ \\
genotype $\times$ treatment & 2 & $2.61^{\text {ns }}$ & $1.47^{\text {ns }}$ & $695.41^{*}$ & $0.0003^{\text {ns }}$ \\
Al concentration $\times$ treatment & 6 & $2.16^{\text {ns }}$ & $1.22^{\text {ns }}$ & $388.22^{*}$ & $0.0002^{\text {ns }}$ \\
genotype $\times$ Al concentration & 3 & $12.96^{\text {ns }}$ & $7.29^{\text {ns }}$ & $1117.79^{* *}$ & $0.001^{\text {ns }}$ \\
genotype Al concentration $\times$ treatment & 6 & $2.23^{\text {ns }}$ & $1.25^{\text {ns }}$ & $441.54^{*}$ & $0.0002^{\text {ns }}$ \\
Error & 43 & 7.00 & 3.94 & 150.00 & 0.0006 \\
CV & & 14.29 & 5.69 & 12.96 & 6.91 \\
\hline
\end{tabular}

CV: Coefficient of variation. $\mathrm{df}=$ Degrees of freedom; ${ }^{* *},{ }^{* *},{ }^{*}$ : significant at $0.001,0.01$ and 0.05 probability level; ns: non significant.

superoxide dismutase activity in Al-sensitive genotypes than that of Al-resistant genotypes under Al treatments. It was thus, indicated that induction or inhibition of specific antioxidant enzymes in plants in response to Al-stress was genotype dependent. We suppose that, induction of antiperoxi-dation enzymes by $\mathrm{Al}$ treatment may contribute to their Al tolerance by a suppression of oxidative damages caused by Al stress. Similar enhanced tolerance to oxidative stress and/or heavy metal stress was also observed by expression of antioxidant enzymes [glutathione S-transferase (GST), superoxide dismutase (SOD), peroxidase and dehydroascorbate reductase] in transgenic plants (Ezaki et al., 2000; Lee et al., 2007).

\section{ACKNOWLEDGEMENTS}

The authors are gratefully thanked to the institute of biotechnology, Urmia University, Iran. The reviewers are kindly acknowledged for their helpful suggestions. The authors are also thankful to Javanan khayer institution in East \& West Azarbayejan, Iran, for their contribution to grant the interest free loan for supporting the scientific research.

\section{Abbreviations}

APX, Ascorbate peroxidase; CAT, catalase; GR, glutathione reductase; SOD, superoxide dismutase; ROS, reactive oxygen species; Al, aluminum; GST, glutathione S-transferase.

\section{REFERENCES}

Allen RD (1995). Dissection of oxidative stress tolerance using transgenic plants. Plant Physiol. 107: 1049-1054.

Asada K (1992). Ascorbate peroxidase-a hydrogen peroxide scavenging enzyme in plant. Physiol. Plant 85: 235-241.

Boscolo PR, Menossi M, Jorge RA (2003). Aluminum-induced oxidative stress in maize. Phytochemistry, 62: 181-189.

Cakmak I, Horst WJ (1991). Effect of aluminum on lipid peroxidation, superoxide dismutase, catalase, and peroxidase activities in root tips of soybean (Glycine max). Physiol. Plant 83: 463-468.

Cruz-Ortega R, Cushman JC, Ownby JD (1997). cDNA clones encoding 
1,3- $\beta$-glucanase, and fimbrin-like cytoskeletal protein are induced by Al toxicity in wheat roots. Plant Physiol. 114:1453-1460.

Darko E, Ambrus H, Stefanovits-Banyai E, Fodor J, Bakos F, Barnabas $\mathrm{B}$ (2004). Aluminium toxicity, Al tolerance and oxidative stress in an Al-sensitive wheat genotype and in Al-tolerant lines developed by in vitro microspore selection. Plant Sci. 166: 583-591.

Desikan R, Neill SJ, Hancock JT (2000). Hydrogen peroxide induced gene expression in Arabidopsis thaliana. Free Radic. Biol. Med. 28:773-778.

Dipierro N, Mondelli D, Paciolla C, Brunetti G, Dipierro S (2005). Changes in the ascorbate system in the response of pumpkin ( Cucurbita pepo L.) roots to aluminium stress. J. Plant Physiol. 162: 529-536.

Ezaki B, Gardner RC, Ezaki Y, Matsumoto H (2000). Expression of aluminum-induced genes in transgenic Arabidopsis plants can ameliorate aluminum stress and/or oxidative stress. Plant Physiol. 657: 665-122.

Ezaki B, Tsugita S, Matsumoto H (1996). Expression of a moderately anionic peroxidase is induced by aluminium treatment in tobacco cells: Possible involvement of peroxidase isozymes in aluminium ion stress. Physio. Plant 96: 21-28.

Ezaki B, Yamamoto Y, Matsumoto $\mathrm{H}$ (1995). Cloning and sequencing of the cDNAs induced by aluminium treatment and $\mathrm{Pi}$ starvation in cultured tobacco cells. Physiol. Plant 93: 11-18.

Guo T, Zhang G, Zhou M, Wu1 F, Chen J (2004). Effects of aluminum and cadmium toxicity on growth and antioxidant enzyme activities of two barley genotypes with different Al resistance. Plant and Soil, 258: 241-248.

Hamilton CA, Good AG, Taylor GJ (2001). Induction of vacuolar ATPase and mitochondrial ATP synthase by aluminum in an aluminum-resistant cultivar of wheat. Plant Physiol. 125: 2068-2077.

Kato M, Shimidzu S (1987). Chlorophyll metabolism in higher plants. VII, chlorophyll degradation in senescing tobacco leaves: phenolicdependent peroxidative degradation. Can. J. Bot. 65: 729-735.

Kuo MC, Kao CH (2003). Aluminum effect on lipid peroxidation and antioxidative enzyme activities in rice leaves. Biologia plantarum, 46: 149-152.

Lee DH, Kim YS, Lee CB (2001). The inductive responses of the antioxidant enzymes by salt stress in the rice (Oryza sativa L.). Plant Physiol. 158: 737-745.

Lee YP, Kim SH, Bang JW, Lee HS, Kwak SS, Kwon SY (2007). Enhanced tolerance to oxidative stress in transgenic tobacco plants expressing three antioxidant enzymes in chloroplasts. Plant Cell 26: 591-598.

Ma B, Wan J, Shen $\mathrm{Z}$ (2007). $\mathrm{H}_{2} \mathrm{O}_{2}$ production and antioxidant responses in seeds and early seedlings of two different rice varieties exposed to aluminum. Plant Growth Regul. 52: 91-100.

Meriga B, Reddy BK, Rao KR, Reddy LA, Kavi Kishor PB (2004). Aluminium-induced production of oxygen radicals, lipid peroxidation and DNA damage in seedlings of rice (Oryza sativa L.). J. Plant Physiol. 161: 63-68.

Mizuno M, Kamei M, Tsuchida $H$ (1988). Ascorbate peroxidase and catalase cooperate for protection against hydrogen peroxide generated in potato tubers during low-temperature storage. Biochem. Mol. Biol Int 44: 717-726.

Nakano Y, Asada K (1981). Hydrogen peroxide is scavenged by ascorbat-specific peroxidase in spinach chloroplasts. Plant Cell Physiol. 22: 867-880.

Neill SJ, Desikan R, Clarke A, Hurst RD, Hancock JT (2002). Hydrogen peroxide and nitric oxide as signaling molecules in plants. J. Exp. Bot. 53: 1237-1247.

Paoliett F, Aldinucci D, Mocali A, Capparini A (1986). A sensitive spectrophotometric method for the determination of superoxide dismutase activity in tissue extracts. Anal. Biochem. 154: 336-541.

Richards KD, Schott EJ, Sharma YK, Davis KR, Gardner RC (1998). Aluminum induces oxidative stress genes in Arabidopsis thaliana. Plant Physiol. 116: 409-418.

Sharma P, Dubey RS (2007). Involvement of oxidative stress and role of antioxidative defense system in growing rice seedlings exposed to toxic concentrations of aluminum. Plant Cell 26: 2027-2038.

Siegel BZ (1993). Plant peroxidases-an organismic perspective. Plant Growth Regul 12: 303-312.
Simonovicova M, Tammas L, Huttova J, Mistrik I (2004). Effect of aluminium on oxidative stress related enzymes activities in barley roots. Biologia Plantarum, 48: 261-266.

Snowden KC, Richards KD, Gardner RC (1995). Aluminum-induced genes. Induction by toxic metals, low calcium, and wounding and pattern of expression in root tips. Plant Physiol. 107: 341-348.

Tamas L, Huttova J, Mistrık I (2003). Inhibition of Al-induced root elongation and enhancement of Al-induced peroxidase activity in Alsensitive and Al-resistant barley cultivars are positively correlated. Plant Soil 250: 193-200.

Tamas L, Simonovicova M, Huttova J, Mistrik I (2004). Aluminium stimulated hydrogen production of germinating barley seeds. Environ. Exp. Bot. 51: 281-288.

Van Breusegem F, Vranova E, Dat JF, Inze D (2001). The role of active oxygen species in plant signal transduction. Plant Sci. 161: 405-414.

von Uexkull HR, Mutert E (1995). Global extent, development and economic impact of acid soils. Kluwer Academic Publishers, Dordrecht, the Netherlands. pp. 5-19.

Wintermans JFGM, De Mots A (1965). Spectrophotometric characteristics of chlorophyll $a$ and $b$ and their pheophytins in ethanol. Biochem. Biophys. Acta. 109: 149-152.

Zakir Hossain AKM, Ohno T, Koyama H, Hara T (2005). Effect of enhanced calcium supply on aluminum toxicity in relation to cell wall properties in the root apex of two wheat cultivars differing in aluminum resistance. Plant and soil, 276: 193-204. 